\title{
Acalvaria in a Malian girl: a new surviving case
}

Lassana Cissé ${ }^{1}$, Salimata Diarra ${ }^{2}$, Abdoulaye Yalcouyé ${ }^{2}$, Youlouza Coulibaly ${ }^{3}$, Abdoulaye Tamega $^{2}$, Alassane Baneye Maiga ${ }^{4}$, Oumou Traoré ${ }^{2}$, Mahamadou Kotioumbé ${ }^{3}$, Moussa Sangaré $^{2}$, Seybou Hassane Diallo ${ }^{5}$, Salimata Diallo ${ }^{5}$, Hamidou O Bah ${ }^{5}$, Assiatou Simaga $^{6}$, Koné Fatogoma Issa ${ }^{5}$, Thomas Coulibaly ${ }^{2}$, Oumar Samassékou ${ }^{7}$, Cheick Guinto ${ }^{2}$, and Guida Landouré2

${ }^{1}$ Centre Hospitalier Universitaire du Point "G"

${ }^{2}$ Centre Hospitalier Universitaire du Point G

${ }^{3}$ Centre Hospitalier Universitaire du Point G

${ }^{4}$ Centre Hospitalier Universitaire du Point "G"

${ }^{5}$ Centre Hospitalier Universitaire Gabriel Touré

${ }^{6}$ Institut d'Ophtalmologie Tropicale de l'Afrique

${ }^{7}$ Universite des Sciences des Techniques et des Technologies de Bamako

November 1, 2021

\begin{abstract}
Acalvaria is a rare and lethal congenital malformation characterized by the absence of the cranial vault bones, dura mater and associated muscles with complete cranial content. We report a 5-year-old Malian girl seen at 20 months old for facial dysmorphia, hemiparesis, and a cranial bone defect that improved progressively.
\end{abstract}

\section{Acalvaria in a Malian girl: a new surviving case}

Cissé $\mathrm{L}^{1}$, Diarra $\mathrm{S}^{1,2}$, Yalcouyé $\mathrm{A}^{1}$, Coulibaly $\mathrm{Y}^{3}$, Taméga $\mathrm{A}^{1}$, Maiga $\mathrm{AB}^{1}$, Traoré $\mathrm{O}^{1}$, Kotioumbé $\mathrm{M}^{1}$, Sangaré $\mathrm{MA}^{1}$, Diallo $\mathrm{SH}^{4,5}$, Diallo $\mathrm{S}^{4}$, Ba $\mathrm{HO}^{5,6}$, Simaga $\mathrm{A}^{5,7}$, Koné $\mathrm{FI}^{8}$, Coulibaly $\mathrm{T}^{1,5}$, Samassékou $\mathrm{O}^{5}$, Guinto $\mathrm{CO}^{1,5}$, Landouré $\mathrm{G}^{1,2,5}$, the H3Africa consortium.

${ }^{1}$ Service de Neurologie, Centre Hospitalier Universitaire du Point G, Bamako, Mali,

${ }^{2}$ Neurogenetics Branch, NINDS, NIH, Bethesda, MD, USA

${ }^{3}$ Service d'Imagerie Médicale, Centre Hospitalier Universitaire du Point G, Bamako, Mali

${ }^{4}$ Service de Neurologie, Centre Hospitalier Universitaire Gabriel Touré, Bamako, Mali

${ }^{5}$ Faculté de Médecine et d'Odontostomatologie, USTTB, Bamako, Mali

${ }^{6}$ Service de Cardiologie, Centre Hospitalier Universitaire Gabriel Touré, Bamako, Mali

${ }^{7}$ Institut d'Ophtalmologie Tropicale de l'Afrique, Bamako, Mali

${ }^{8}$ Service d'ORL, Centre Hospitalier Universitaire Gabriel Touré, Bamako, Mali

\section{Corresponding author:}

Lassana CISSE, Service de Neurologie, Centre Hospitalier Universitaire du Point G, Bamako, Mali, Email : lassciss@gmail.com 


\begin{abstract}
Acalvaria is a rare and lethal congenital malformation characterized by the absence of the cranial vault bones, dura mater and associated muscles with complete cranial content. We report a 5-year-old Malian girl seen at 20 months old for facial dysmorphia, hemiparesis, and a cranial bone defect that improved progressively.
\end{abstract}

Keywords: Acalvaria, congenital malformation, Mali, Africa.

\title{
Key Clinical Message
}

Acalvaria is a rare lethal congenital malformation with only a few surviving cases described worldwide. We report a 5-year-old Malian girl with acalvaria seen at 20 months old for hemiparesis and had partial absence of the frontal and parietal bones.

\section{INTRODUCTION}

Acalvaria is a rare congenital malformation characterized by the absence of the cranial vault bones, the dura mater and associated muscles. ${ }^{1}$ Although the basal and facial bones are normally developed, the cranial content can often be abnormal. ${ }^{1-3}$ Its pathogenesis is not well known, but the abnormality occurring after neurulation is the most accepted theory. ${ }^{1-3}$ It is a fatal condition with only a few cases of survivors often with severe neurological disorders. ${ }^{1-4}$ To date, only one case has been reported in Africa. ${ }^{5}$ We report a new surviving case presenting with facial dysmorphia and hemiparesis.

\section{CASE REPORT}

A 20-month-old girl was seen in our department for right body side muscle weakness. She was born of a normal pregnancy and a non-incidental birth. The obstetrical ultrasounds of the three trimesters revealed no abnormalities. There is no consanguinity between the parents and the patient is the third of a sibship with two healthy sisters. Her 31-years-old mother did not report any past medical history of disease, neither teratogenic drugs intake.

The mother noticed at birth a strange softness of the whole upper part of the head and was reassured by the healthcare providers that time will address the issue as the child grows up. Four months later, she developed muscle weakness in the right side body, and started walking at 10 months age. Her pediatrician referred her to a rehabilitation center where she underwent physical therapy.

With the persisting hemiparesis, parents decided to come to our clinic for a better management. In clinical examination she weighted $9 \mathrm{~kg}$ with a $43 \mathrm{~cm}$ head circumference, and a facial deformity characterized by a frontal depression. The upper parietal and frontal posterior region measuring $21 \mathrm{x} 15 \mathrm{~cm}$ was soft but completely recovered with healthy skin and hair. She was cooperative and did not have a language or behavioral disorder. There was a right hemiparesis with a motor strength rated $3 / 5$ in upper and lower limbs, but walked without assistance. The tendon reflexes were brisk in the right side and the Babinski sign was present. There were no other neurological anomalies as well as auditory or visual disturbance. Ophthalmological and Ear Nose and Throat (ENT) examinations were also normal. In addition, cardiac and abdominal ultrasounds were normal. Blood chemistries including total cell count, serum creatinine, alkaline phosphatase and ions were normal except phosphorus that was slightly elevated at $1.84 \mathrm{mmol} / \mathrm{l}$ (normal range $0.87-1.50 \mathrm{mmol} / \mathrm{l}$ ). Brain CT-scan revealed a dilatation of the occipital horns of the lateral ventricles that persisted after three years of evolution (Figure 1A \& B). The three dimensional CT (3D CT) reconstruction of the skull bones showed the absence of the posterior part of the frontal and the upper part of the parietal bones with normal facial bones and the skull base (Figure 2A \& B). Three years later a reduction of the soft tissue from $21 \times 15 \mathrm{~cm}$ to $14.5 \times 10 \mathrm{~cm}$ was noticed with a notable progression of bone development (Figure $2 \mathrm{C}$ \& D). Parents were reassured about the possible bone development progression but were advised to protect the patient from activities that may lead head injury and a follow up every six months. The course of the disease was marked by two episodes of generalized tonic and clonic seizures in three years interval. The first episode occurred in a febrile context related to malaria infection and the electroencephalogram was normal. Therefore, we decided a therapeutic abstention. No other abnormalities were noted. 


\section{DISCUSSION}

Acalvaria is an extremely rare congenital malformation involving the cranial vault bones, the dura matter and associated muscles. The development of the facial and the base bones is normal with a complete cranial content. ${ }^{1-3}$ However association with other abnormalities is often reported, including hydrocephalus, holoprosencephaly, micropolygyria, encephalocele or amniotic band syndrome resulting premature death, most often prenatally. ${ }^{1-6}$ The few survival cases deal with a mental retardation but with motor weakness or seizure has not been reported.

This is the second case reported in Africa so far after the one from South Africa who was a 6-month of age girl presenting head softness with no other clinical symptoms but brain CT-scan showed the absence of major skull bones with normal facial and skull base bones. However, no follow-up data beyond the 6 months were given. ${ }^{5}$

The patient presented focal neurological deficit and seizures but no intellectual disability. Brain CT-scan showed an absence of the upper part of the frontal and the parietal bones and a dilatation of the occipital horns of the lateral ventricles which may be related to this region's atrophy. Although, the lesions found on the brain CT-scan didn't correlate with the clinical findings, brain malformations or other brain lesions from perinatal brain related trauma that went unnoticed could explain these symptoms. Antenatal diagnosis is possible from 11 weeks of amenorrhea with obstetrical ultrasound; but an elevated Alpha foetoprotein and non-detected unconjugated estradiol could help with the diagnosis. ${ }^{2,7,8}$ However, this patient was diagnosed very lately. An unusual longevity that could be due to mild brain abnormalities or yet to identify genetic or environmental factors. This presentation should be differentiated from anencephaly, exencephaly and acrania.

Anencephaly differs from acalvaria as it presents with a total or partial absence of the brain and scalp..$^{8,9}$ Exencephaly is considered as precursor to anencephaly. ${ }^{8,9}$ Acrania is often used as the synonym of acalvaria but in this condition, the absence of the skull bones may extend beyond the vault bones and is often accompanied by anencephaly. ${ }^{8,10}$

The pathophysiology of acalvaria is still unclear but a deficit in the mesenchymal migration is the most accepted hypothesis. ${ }^{1-3}$ Normally, mesenchymal migration occurs after the neural tube closure around the fourth week of the embryonic development. ${ }^{2,3,11}$ This lack of migration would explain the absence of bones and muscles while the brain tissue and the skin of ectodermal origin are present. ${ }^{2-3}$ However, some authors argued that this could be a variant of the anomalies linked to the neural tube closure defect. ${ }^{2}$ The etiology of acalvaria is not identified but a female predominance is reported in the literature as the case we report here. $^{2}$ To date, there is no consensus on its treatment which remains largely conservative consisting of parents' reassurance, monitoring and symptomatic treatment of any associated condition. ${ }^{2,3,5}$ As in the present study, continued bone development has been observed in other conditions with cranial vault absence such as Aplasia cutis congenita. ${ }^{12}$ It has therefore been suggested surgical treatment including bone grafting or plastic surgery should be reserved for school age children. ${ }^{5,12}$ An early diagnosis could help to limit the complications, especially the antenatal ones with a good planning of the delivery.

\section{CONCLUSION}

Acalvaria is a rare fatal congenital malformation with only a few surviving cases described worldwide. We report a new surviving case with facial dysmorphia and right hemiparesis whose diagnosis was delayed. The improvement of life quality of these patients relies on the improvement of the antenatal diagnosis allowing a good planning of the delivery in order to prevent the disability linked to a probable perinatal suffering. Reporting cases of this disease allows scientists to deepen their understanding in order to find a better therapeutic strategy for these children with a vulnerable brain.

\section{CONFLICT OF INTEREST}

The authors declare no $\mathbf{c}$ onflict of interest.

\section{ACKNOWLEDGEMENTS}


This work has been supported by the funds U01HG007044 from the National Institute of Neurological Disorders and Stroke, USA, administered by the National Human Genome Research Institute as part of the H3Africa initiative.

\section{AUTHOR CONTRIBUTIONS}

LC, GL, COG: conception and design of the study, data interpretation, drafting of the manuscript, final approval of the version to be submitted, submission of the manuscript, agreed to be accountable for all aspects of the work. S Diarra, AY, YC, AT, ABM, OT, MK, MAS, SHD, S Diallo, HOB, AS, FK, TC, OS: data interpretation, critical revising of the manuscript, final approval of the version to be submitted and agreed to be accountable for all aspects of the work.

\section{ETHICS STATEMENT}

Study is approved by the institutional Ethical Committee of Faculté de Médecine et d'Odonto-Stomatologie (FMOS), Mali. The patient's Mother signed the informed consent forms for data sharing including photos and CT-scan.

\section{DATA AVAILABILITY STATEMENT}

Data that support the findings of this study are available from the corresponding author upon reasonable request

\section{REFERENCES}

1. Harris C. P, Townsend J. J, Carey J. C. 1993. Acalvaria: a unique congenital anomaly. Am J Med Genet 46 :694-699. https://doi.org/10.1002/ajmg.1320460620.

2. Khadilkar V.V, Khadilar A.V, Nimbalkar A. A, Kinnare A. S, 2004. Acalvaria. Indian Pediatr 41 :618-612.

3. Gupta V, and Kumar S. 2012. Acalvaria: A rare congenital malformation.J Pediatr Neurosci 7 (3):185187. https://doi.org/10.4103/1817-1745.106474.

4. Kurata H, Tamaki N, Sawa H, Oi S, Katayama K, Mochizuki M, et al. 1996. Acrania: Report of the first surviving case. Pediatr Neurosurg 24 :52-54. https://doi.org/10.1159/000121015.

5. Ouma J. R. 2019. Acalvaria - report of a case and discussion of the Literature. Br J Neurosurg 33 (2):224-225. https://doi.org/10.1080/02688697.2017.1322685.

6. Chandran S, Lim M.K, Yu V.Y. 2000. Fetal acalvaria with amniotic band syndrome. Arch Dis Child 82 (1):F11-F13, https//doi.org/10.1136/fn.82.1.f11.

7. Karasu Y, Bozkurt M, Gençdal S, G. Tanas G, Dede S, Yumru A.E, et al. 2015. Prenatal diagnosis of fetal acrania using two and three dimensional ultrasound. Proceedings in Obstetrics and Gynecology5(1): Article 4 [ 6 p.]. Available from: http://ir.uiowa.edu/poghttps://doi.org/10.17077/2154-4751.1281.

8. Santana E. F. M, Araujo Júnior E, Tonni G, Da Silva Costa F, Meagher S. 2018. Acraniaexencephaly-anencephaly sequence phenotypic characterization using two- and three-dimensional ultrasound between 11 and 13 weeks and 6 days of gestation. J Ultrason 18 (74):240-246. https://doi.org/10.15557/JoU.2018.0035.

9. Stumpf D. A. et al. 1990. The infant with Anencephaly. $N$ Engl J Med 322 (10):669-674. https://doi.org/10.1056/NEJM199003083221006.

10. Sharif A, and Zhou Y. 2016. Fetal MRI Characteristics of Exencephaly: A Case Report and Literature Review. Case Rep Radiol4 :1-4. https://doi.org/10.1155/2016/9801267

11. Ferguson J. W, Atit R. P. 2019. A tale of two cities: The genetic mechanisms governing calvarial bone development. Genesis57 (1): e23248. https://doi.org/10.1002/dvg.23248

12. Bang R. L, Ghoneim I. E, Gang R. K, Najjadah I. A1. 2003. Treatment dilemma: Conservative versus surgery in cutis aplasia congenita. Eur J Pediatr Surg 13 (2):125-129. https://doi.org/10.1055/s-200339562

Figure legends: 
Figure 1 : Brain CT-scan of the patient. A) Shows a dilatation of the occipital horns of the lateral ventricles at the time of diagnosis, B) Shows the persistence of the dilatation of the occipital horns of the lateral ventricles after three years.

Figure 2: CT-scan of the skull in 3D reconstruction; A) anterior view showing the absence of the upper part of the frontal and the parietal bones, B) posterior view showing the absence of the parietal bone with preservation of the occipital bone. C) and D) progression of the cranial bone development after three years of evolution with a notable reduction of the bone defect.
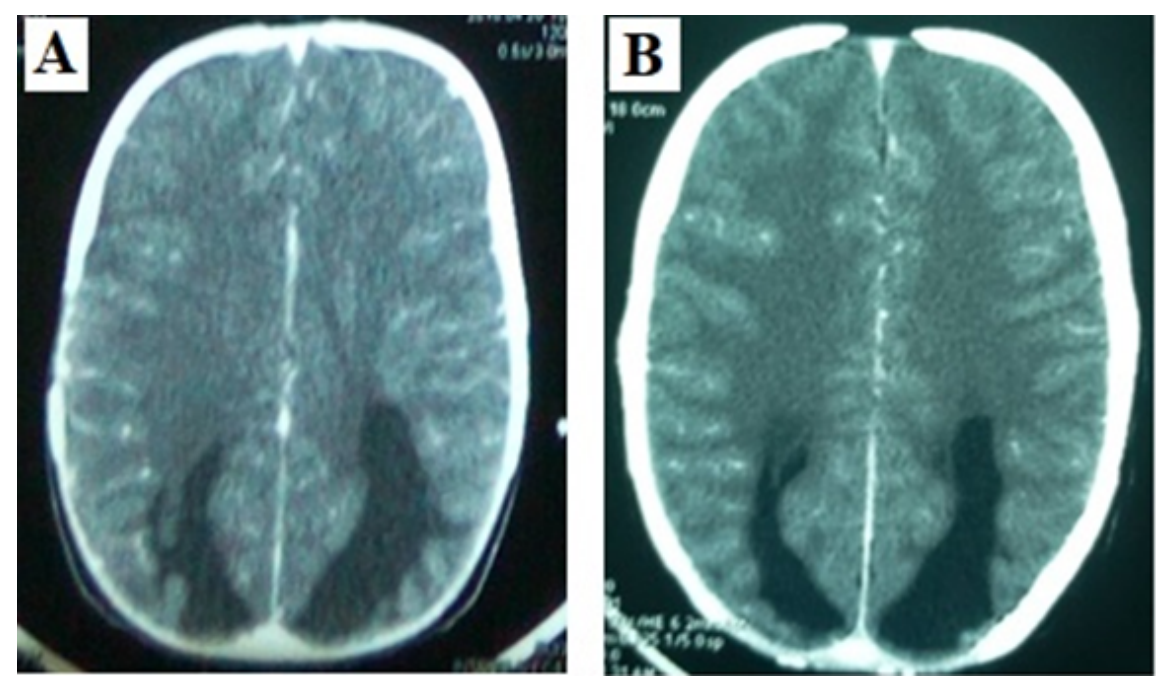

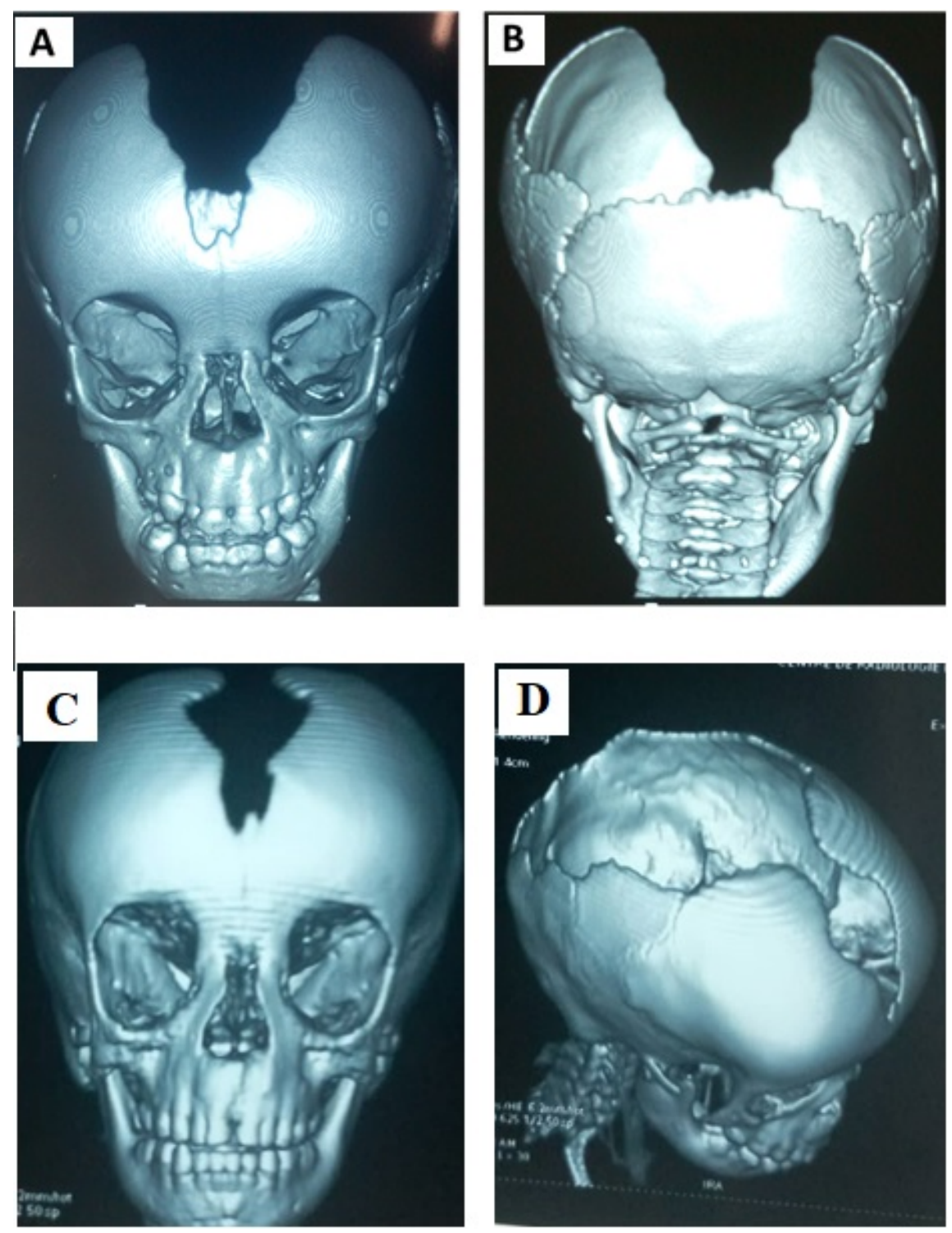